\title{
MANEJO LAPAROSCÓPICO DE LA ENFERMEDAD PÉLVICA INFLAMATORIA SEVERA: REPORTE DE TRES CASOS Y REVISIÓN DE LA LITERATURA
}

\section{Laparoscopic management of severe pelvic inflammatory disease: a report of three cases and a literature review}

Gustavo Adolfo Calle-Gómez, M.D.*, Adriana María Arango-Martínez, M.D.**, Juan David Castañeda-Roldán, M.D. **, José Fernando De los Ríos-Posada, M.D.**, Eduardo Serna, M.D.**, Ricardo Vásquez, M.D.**, Luis Alberto Jiménez-Zuluaga, M.D.***, Antonio González, M.D.***

Recibido: junio 10/10 - Aceptado: junio 7/11

\section{RESUMEN}

Objetivo: hacer una revisión de la literatura sobre la utilidad de la laparoscopia en el tratamiento del absceso tubovárico en términos de mejoría, estancia y fertilidad posterior.

Materiales y métodos: se presentan tres casos de pacientes con enfermedad pélvica inflamatoria severa que consultaron a una institución privada de referencia ubicada en Medellín (Colombia), a quienes se les practicó una laparoscopia operatoria, además de la administración de un antibiótico parenteral como manejo. Se realizó una búsqueda en la base de datos Medline vía PubMed con las palabras clave: "tubo-ovarian abscess, treatment, laparoscopy, fertility”. Se realizó además una búsqueda de textos clásicos y guías de manejo.

\footnotetext{
* Especialista en Cirugía Endoscópica Ginecológica, Ginecólogo y Obstetra. Unidad de Cirugía Endoscópica Ginecológica. Clínica del Prado. Posgrado Cirugía Endoscópica Ginecológica. Universidad CES. Medellín (Colombia). Correo electrónico: gustavocalle.md@gmail.com

** Especialista en Cirugía Endoscópica Ginecológica, Ginecólogo y Obstetra. Docente de Cirugía Endoscópica Ginecológica. Universidad CES. Medellín (Colombia).

*** Fellow en Cirugía Endoscópica Ginecológica. Universidad CES. Medellín (Colombia).
}

Resultados: se encontraron 49 artículos de los cuales 13 eran relevantes al problema en cuestión. De estas publicaciones, 8 eran artículos de revisión, 4 series de casos, y 1 un estudio de cohortes retrospectivas. Conclusión: el manejo laparoscópico conservador del absceso tubovárico en el período inicial de la enfermedad, adicional a los antibióticos, surge como una alternativa que podría mejorar los resultados a corto y largo plazo. Se requieren estudios de alta calidad que respalden esta observación.

Palabras clave: enfermedad pélvica inflamatoria, absceso pélvico, absceso tubovárico, laparoscopia.

\section{SUMMARY}

Objective: carrying out a literature review about the usefulness of laparoscopy in treating tuboovarian abscesses in terms of improvement, hospital stay and subsequent fertility.

Materials and methods: three cases of patients suffering from severe pelvic inflammatory disease are presented; they consulted at a private reference institution in Medellín, Colombia, and were then submitted to laparoscopy, accompanied by parenteral antibiotics as management. A search was made in the Medline database via PubMed using 
the following key words: "tubo-ovarian abscess, treatment, laparoscopy, fertility." A search was also made in classical texts on the subject and management guidelines.

Results: 49 pertinent articles were found, of which 13 were relevant to the matter in hand; 8 were review articles, 4 were case-series and 1 was a retrospective cohort study.

Conclusion: conservative laparoscopic management of tubo-ovarian abscess during the disease's initial period, accompanied by the appropriate antibiotic regime, emerged as an alternative which could improve short- and long-term results. High-quality studies are thus needed to confirm such observation.

Key words: pelvic inflammatory disease, pelvic abscess, tubo-ovarian abscess, laparoscopy.

\section{INTRODUCCIÓN}

La Enfermedad Pélvica Inflamatoria (EPI) se define como la infección del tracto genital femenino superior, que incluye cualquier combinación de endometritis, salpingitis, absceso tubovárico y peritonitis pélvica. ${ }^{1}$ La incidencia y la prevalencia de la EPI son difíciles de establecer, entre otras razones por la diversidad en los criterios diagnósticos, la baja especificidad en los criterios clínicos, o la presentación silenciosa de la enfermedad. ${ }^{2}$ En Estados Unidos se calcula que cerca de un millón de pacientes son tratadas anualmente por EPI, de las cuales 200000 requieren hospitalización. ${ }^{3}$ En el Instituto Materno Infantil de Bogotá (Colombia) la prevalencia se ha estimado en 9,2 por 1000 mujeres fértiles. ${ }^{4}$ El absceso tubovárico (ATO), una de las formas del absceso pélvico, se presenta como uno de los cuadros clínicos más graves de la EPI, y puede ocurrir hasta en un 34\% de las pacientes con la afección, ${ }^{5}$ aunque en Chile se ha reportado que puede presentarse hasta en el 74\% de las pacientes. ${ }^{6}$

Se sabe que las secuelas de la EPI y del ATO son entre otras, la infertilidad, el embarazo ectópico y el dolor pélvico crónico. ${ }^{7}$ El manejo universalmente aceptado para el ATO ha sido la terapia con antibióticos, reservando el manejo quirúrgico para las pacientes que no responden al manejo médico o cuando hay sospecha de ruptura del absceso. ${ }^{8}$ Aunque la laparoscopia ha sido considerada como el patrón de oro para el diagnóstico de la EPI, es una herramienta costosa y no siempre accesible en los centros de atención médica, por lo tanto su uso en la EPI no complicada se reserva para pacientes con diagnóstico dudoso. ${ }^{4}$ No obstante, algunos autores proponen un drenaje temprano del absceso con el fin de mejorar el pronóstico reproductivo y disminuir la posibilidad de dolor pélvico crónico. En el presente artículo se presentan 3 casos de pacientes con absceso pélvico quienes fueron manejadas por laparoscopia en la Unidad de Cirugía Endoscópica Ginecológica de la Clínica del Prado de Medellín (Colombia), con el objetivo de hacer una revisión de la literatura acerca del papel de la laparoscopia en el tratamiento del absceso tubovárico en términos de mejoría, estancia y fertilidad posterior.

\section{REPORTE DE CASOS}

\section{Caso 1}

Paciente de 34 años de edad, soltera, quien acudió al servicio de urgencias por presentar un cuadro clínico de 5 días de evolución de fiebre alta no cuantificada, cefalea y dolor abdominal de predominio en flanco izquierdo. La paciente es G2P1A1V1, con antecedente de un legrado por aborto de 8 semanas de gestación que se le había practicado 5 días antes del último ingreso institucional. La paciente fue usuaria de DIU hasta el momento del legrado. Al momento del examen se encuentra normotensa, con temperatura de $38,8^{\circ} \mathrm{C}$, pulso de 98 latidos por minuto. Se halló además, que la vagina de la paciente estaba hipertérmica, con mucho dolor a la movilización del útero y con marcada resistencia muscular. El hemograma mostró hemoglobina de 8,9, leucocitos de 25570 , neutrófilos 88\%, proteína C reactiva $198 \mathrm{mg} / \mathrm{L}$. La ecografía transvaginal evidenció un útero de $77 \times 53 \times 52 \mathrm{~mm}$ con el endometrio de 3,7 mm. En anexo izquierdo se observa imagen ecomixta compatible con complejo tubovárico de 59x47x41 mm. 
Se inició el tratamiento con clindamicina $600 \mathrm{mg}$ IV cada 6 horas y gentamicina $240 \mathrm{mg}$ IV cada 24 horas; y 36 horas después fue llevada a laparoscopia. Se encontró absceso tubovárico bilateral y múltiples adherencias pélvicas. Se realizó una lisis de adherencias, drenaje por aspiración y lavado de cavidad peritoneal con 4000 cc de solución salina. Se tomaron muestras para cultivos cuyos reportes fueron negativos. La evolución posquirúrgica fue satisfactoria y a la paciente se le dio de alta al continuar afebril por 48 horas con manejo de antibiótico ambulatorio hasta completar 14 días.

\section{Caso 2}

Paciente de 42 años de edad, con un diagnóstico de miomatosis uterina sintomática confirmada por ecografía. Se programó para histerectomía laparoscópica. En el acto operatorio se halló abundante cantidad de pus en la pelvis con síndrome adherencial severo. Se decide no llevar a cabo la histerectomía planeada y a cambio se realiza drenaje del absceso con lavado exhaustivo. Se le ordenó a la paciente un tratamiento con clindamicina $600 \mathrm{mg}$ IV cada 6 horas, gentamicina $160 \mathrm{mg}$ IV cada 24 horas y doxiciclina $100 \mathrm{mg}$ vía oral cada 12 horas. Paraclínicos: hemograma: Hb: 10,9 g; leucocitos: 13 660; neutrófilos: 76,8\%; proteína C reactiva: 24,7 mg/dL; cultivo de líquido peritoneal: negativo; cultivo para gonorrhoeae: negativo; BK: negativo; serología para lúes: negativa; HIV: negativo; antígeno de superficie para hepatitis B: negativo. La paciente recibió tratamiento intrahospitalario por 3 días y posteriormente fue dada de alta con tratamiento ambulatorio hasta completar 14 días. Paraclínicos de control postratamiento: hemograma: Hb: 11,6 g/dL; leucocitos: 5910; neutrófilos: 50,6\%; proteína C reactiva: $2,1 \mathrm{mg} / \mathrm{dL}$.

La paciente fue llevada a una laparoscopia nuevamente, en donde se hallló un útero con tamaño aumentado por la miomatosis, síndrome adherencial severo que compromete ambos anexos, colon sigmoide y recto; oclusión del fondo del saco de Douglas, retracción de los ligamentos uterosacros, trompas uterinas edematosas y dilatadas, ovario derecho edematizado y firmemente adherido al ligamento uterosacro. Se practicó una histerectomía laparoscópica total, salpingectomía bilateral y ooforectomía derecha. El útero pesó $370 \mathrm{~g}$. El cultivo de secreción peritoneal fue positivo para Chlamydia trachomatis. En el período posoperatorio se le formuló ceftriaxona, clindamicina y doxiciclina. A los 3 días se le dio de alta con doxiciclina hasta completar 14 días y se ordenó tratamiento para la pareja. La paciente fue evaluada una semana después y luego al mes con una evolución satisfactoria.

\section{Caso 3}

Paciente de 48 años de edad quien consultó por cuadro de 5 días de evolución de dolor pélvico y fiebre no cuantificada. La ecografía muestra imagen anexial derecha compatible con absceso tubovárico. El hemograma mostró 11500 leucocitos y la proteína C reactiva es de $5 \mathrm{mg} / \mathrm{dL}$. Se practicó una laparoscopia, donde se halló útero de $8 \mathrm{~cm}$, trompas dilatadas, tortuosas, con abundante secreción purulenta; también hay material purulento en la cavidad pélvica y material fibrinopurulento interasas. Colon sigmoide adherido al fondo uterino y apéndice cecal adherido al anexo. No se evidenció algún compromiso del parénquima de los ovarios. Se practicó por vía laparoscópica: drenaje y lavado del material purulento, adhesiolisis, salpingectomía bilateral.

Se le ordenó un tratamiento con clindamicina 900 mg IV cada 6 horas, gentamicina $240 \mathrm{mg}$ IV cada 24 horas y penicilina cristalina 5 millones de unidades IV cada 6 horas. La paciente recibió tratamiento intrahospitalario por 3 días con excelente evolución. Se le dio de alta con manejo ambulatrio. El control clínico un mes después fue satisfactorio.

\section{MATERIALES Y MÉTODOS Búsqueda bibliográfica y revisión de la literatura}

Se realizó una búsqueda en la base de datos Medline vía PubMed con las palabras clave: "tubo-ovarian abscess, 
treatment, laparoscopy, fertility”. No hubo restricción por idioma o tiempo. Además, se realizó una estrategia en bola de nieve a partir de los artículos seleccionados. También se llevó a cabo una búsqueda de textos clásicos y guías de manejo.

\section{RESULTADOS}

Se encontraron 49 artículos, de los cuales 13 eran relevantes al problema en cuestión. 8 de ellos eran artículos de revisión, 4 series de casos, 1 un estudio de dos cohortes retrospectivas que compararon la laparoscopia con la laparotomía.

\section{DISCUSIÓN}

El absceso pélvico puede tener variedad de etiologías. La mayoría de los casos son abscesos tubováricos secundarios a infecciones de transmisión sexual (ITS) ${ }^{8}$ pero también pueden ocurrir como complicaciones del embarazo, ${ }^{9}$ por endometritis crónica anaeróbica ${ }^{10}$ asociado al uso prolongado del dispositivo intrauterino (DIU) ${ }^{11}$ apendicitis perforada, diverticulitis, o por complicaciones de cirugías pélvicas o abdominales. ${ }^{9}$ El absceso tubovárico corresponde a la EPI severa tanto en la clasificación clínica como en la laparoscópica. ${ }^{12}$

El manejo quirúrgico de la EPI ha evolucionado drásticamente en las últimas décadas. En 1964, Pedowitz y Bloomfield hallaron ruptura del absceso en $16 \%$ de las pacientes con cirugía conservadora previa (24/143); y en 35\% (6/17) de las pacientes a quienes se les conservó al menos un anexo se requirió una histerectomía posteriormente; ${ }^{13}$ por lo tanto, el manejo recomendado, en ese entonces, era la histerectomía abdominal total más salpingooforectomía bilateral. ${ }^{14}$ Pronto empezaron a surgir las desventajas de este manejo radical: $17 \%$ de lesiones en intestino o recto, ${ }^{15}$ además de las consecuencias irreversibles sobre la fertilidad y las derivadas de la menopausia precoz.

Con el advenimiento de nuevos esquemas de terapia antibiótica y mejores medidas de soporte, fue posible realizar un manejo quirúrgico conservando la capacidad reproductiva y sin hallar mayor morbilidad que con la cirugía radical. ${ }^{16}$ Las tasas de embarazo después del manejo con antibióticos no son reportadas en forma consistente en los estudios, pero en las series en donde se reportan varían entre $3,8 \%$ y $15,3 \%$ en las pacientes a quienes se les ha hecho seguimiento. ${ }^{15}$

Por otra parte, los estudios que reportan las tasas de embarazo posterior al manejo quirúrgico conservador son pocos y con un número reducido de pacientes. Landers y Sweet informan del seguimiento a 19 pacientes a quienes se les practicó anexectomía unilateral: hubo 3 embarazos posteriores $(15,8 \%)$ de los cuales uno se trató de un embarazo ectópico. ${ }^{17}$

Con respecto al uso de la laparoscopia en el tratamiento del absceso tubovárico, Dellenbach y colaboradores, en 1972, fueron los primeros en proponer el manejo laparoscópico de los abscesos pélvicos. ${ }^{18}$ Se ha sugerido que el manejo laparoscópico debe ser lo más conservador posible: lisis de las adherencias (acuadisección), drenaje del absceso (con toma de cultivos), excisión de los tejidos infectados o necróticos, e irrigación de la cavidad peritoneal. ${ }^{19}$

Henry-Suchet y su grupo de trabajo publicaron una serie de 50 pacientes a quienes se les realizó manejo laparoscópico dentro de las 24 horas de admisión. A 32 pacientes con absceso reciente (menos de 3 semanas de evolución desde el comienzo de los síntomas), se les practicó drenaje, adhesiolisis (acuadisección), irrigación con una mezcla que contenía solución salina, más antiséptico sumado a un antibiótico (doxiciclina o minociclina). En 18 pacientes el manejo consistió en punción del absceso y drenaje, e irrigación con la misma mezcla que en el caso anterior, pero no se hizo ningún intento de adhesiolisis. No se dejó ningún dren. Todas las pacientes recibieron además tratamiento antibiótico parenteral. Se halló desaparición de la masa en 45 pacientes (90\%). La estancia hospitalaria se redujo de un rango de 15 a 20 días antes del uso de la laparoscopia, a 6 a 8 días. No se reportó ninguna complicación secundaria a la laparoscopia. En las 5 pacientes 
restantes fue necesario practicar laparotomía: en 2 pacientes se realizó salpingooforectomía unilateral, en otras 2 se hizo salpingooforectomía bilateral y en la paciente restante se practicó histerectomía abdominal total más salpingooforectomía bilateral. Se realizó seguimiento a 44 pacientes. 6 pacientes (13\%), tuvieron dolor pélvico persistente. A 25 pacientes se les hizo laparoscopia de segunda mirada hallando: ninguna enfermedad residual en 12 pacientes, adherencias unilaterales en 5 pacientes, y adherencias bilaterales con obstrucción tubárica en 8 pacientes. Entre las pacientes con absceso bilateral reciente (menor de 3 semanas), se les hizo laparoscopia de seguimiento a 7. A 5 de ellas se les encontró anexos normales y 2 tenían adherencias mínimas. 3 de estas pacientes resultaron embarazadas. Entre las pacientes con absceso bilateral de larga evolución (mayor de 3 semanas ) no hubo ningún embarazo. ${ }^{20}$ Esta misma autora publica los resultados de 80 pacientes con tratamiento quirúrgico del ATO por laparoscopia. Pudo hacer seguimiento en 66 hallando dolor pélvico crónico en $22 \%$ de las pacientes quienes tenían absceso de larga evolución (6/28), comparado con 11\% (4/38) de las pacientes con absceso reciente. De éstas 66 pacientes se le hizo laparoscopia de seguimiento a 37, hallando adherencias con obstrucción tubárica en 95\% de los casos con ATO de más de 3 semanas de evolución (15/16), comparado con 15\% (3/21) entre las pacientes con ATO reciente $(\mathrm{p}=0,0001)$. La evaluación de la fertilidad entre 21 pacientes de este estudio que deseaban embarazo halló que 9 pacientes de 15 en el grupo de absceso reciente logró embarazo sin tratamiento (60\%); 4 pacientes adicionales lograron embarazo después de tuboplastia, resultando un porcentaje total de embarazo en este grupo de 86\%. Por el contrario, entre las pacientes con ATO de larga evolución que deseaban embarazo, solo una de 6 pacientes lo logró después de tuboplastia (16\%), diferencia estadísticamente significativa $(\mathrm{p}=0,05) .{ }^{21}$

Reich y colaboradores presentan en su publicación que el manejo laparoscópico conservador entre 25 pacientes con ATO, refiere a una respuesta completa en 24 pacientes, de las cuales 8 lograron embarazo. En 5 casos se realizó laparoscopia de control que mostró adherencias mínimas. ${ }^{22}$

Raiga et al presentan una serie de 39 casos con absceso anexial tratados con laparoscopia. Informan que no se requirió una nueva operación en ningún caso. Realizaron laparoscopia de segunda mirada en 35 de ellas entre 3 y 6 meses después de la cirugía inicial. Debieron realizar adhesiolisis en todos los casos. Realizaron tuboplastia en 17. Refieren que 12 de 19 pacientes que no planificaron tuvieron embarazo espontáneo y 6 pacientes requirieron fertilización in vitro posteriormente. ${ }^{23}$

También se han hecho estudios respecto al manejo más adecuado por laparoscopia: se reclutaron 60 pacientes entre 1994 y 1998; en las pacientes sin interés reproductivo $(\mathrm{n}=35)$ se realizó salpingectomía o salpingooforectomía laparoscópica más antibióticos; entre las pacientes con interés reproductivo $(n=25)$ se realizó drenaje y lavado laparoscópico más antibióticos. Entre el grupo con manejo ablativo se reportaron 9 complicaciones intraoperatorias y 9 complicaciones posoperatorias (18/35: 51\%). Las complicaciones fueron: una perforación intestinal que requirió laparotomía, 4 lesiones de la serosa intestinal, 2 lesiones del omento mayor, 2 laceraciones de colaterales de la arteria ilíaca interna, una paciente con fiebre mayor de $38^{\circ} \mathrm{C}$ por 2 días, 2 pacientes con obstrucción intestinal, una paciente con trombosis del miembro superior, una con trombosis del miembro inferior, y 4 readmisiones por dolor pélvico. Entre las pacientes con manejo conservador no hubo ninguna complicación intraoperatoria y sólo una paciente fue readmitida 2 semanas después de la cirugía por dolor pélvico. A 40 pacientes se les hizo seguimiento telefónico y se registraron 3 embarazos (con 3 nacidos vivos) de 16 pacientes con manejo conservador (18,75\%), mientras que de las 24 pacientes con manejo ablativo solo se reportó 1 embarazo (4,16\%). Los autores concluyen que el manejo quirúrgico debe ser conservador independiente del interés reproductivo de la paciente dada la menor morbilidad. ${ }^{24}$ 
En cuanto al manejo laparoscópico comparado con la laparotomía podemos citar un estudio retrospectivo con 56 pacientes reclutadas entre 1992 y 2000. A 19 pacientes se les practicó laparoscopia entrando con técnica abierta; se tomaron cultivos, se practicó drenaje del absceso, adhesiolisis, escisión del tejido necrótico y lavado con solución de Ringer más heparina al 1 x 1000; se dejó tubo de drenaje por 24-48 horas. A 37 pacientes se les practicó laparotomía y se siguieron los mismos pasos que en el grupo anterior. Todas las pacientes recibieron clindamicina más gentamicina. Las pacientes en el grupo de laparoscopia presentaron menor dolor posoperatorio, más rápida recuperación y un retorno más temprano a sus actividades normales. También presentaron menor morbilidad febril. Los autores concluyen que la laparoscopia es una buena alternativa en el manejo temprano del ATO. ${ }^{25}$

\section{CONCLUSIÓN}

El manejo laparoscópico conservador del absceso tubovárico en el período inicial de la enfermedad, adicional a los antibióticos, parece ofrecer las ventajas de un diagnóstico más preciso, más rápida respuesta, menor tiempo de hospitalización y menores secuelas en cuanto a infertilidad y dolor pélvico crónico. Se deberán realizar experimentos clínicos controlados que comparen el manejo con laparoscopia temprana en el manejo usual con antibióticos o con el drenaje guiado por ultrasonido, tomando en cuenta desenlaces a largo plazo tales como infertilidad y dolor pélvico para tener mayor evidencia al respecto.

\section{AGRADECIMIENTOS}

A todo el personal de la Clínica del Prado en la ciudad de Medellín y a la Universidad CES.

\section{REFERENCIAS}

1. Centers for Disease Control and Prevention (CDC). Pelvic Inflammatory Disease. Visitado 2011
Abr 12. Disponible en: http://www.cdc.gov/std/ treatment/2010/pid.htm

2. Agudelo A, Montoya JG. Enfermedad pélvica Inflamatoria. En: Agudelo A, Montoya JG, editores. Infecciones propias de la mujer. Cali, Colombia: Feriva; p. 69-92.

3. Walker CK, Landers DV. Pelvic abscesses: new trends in management. Obstet Gynecol Surv 1991;46:615-24.

4. Gaitán H, Angel E, Medina M, de Vargas CI, Castañeda E. Diagnóstico de la enfermedad pélvica inflamatoria aguda y sus complicaciones en el Instituto Materno Infantil (IMI) de Santa Fé de Bogotá entre 19921993. Revista de la Facultad de Medicina Universidad Nacional de Colombia 1996;44:134-41.

5. Landers DV, Sweet RL. Current trends in the diagnosis and treatment of tuboovarian abscess. Am J Obstet Gynecol 1985;151:1098-110.

6. Ovalle A, Casanova A, Kakarieka E, de Jourdan F, Salgado K. Epidemiología, resultados clínicos y costos del tratamiento del absceso tubovárico, en un hospital público de Santiago. Rev Chil Obstet Ginecol 2008;73:374-80.

7. Lareau SM, Beigi RH. Pelvic inflammatory disease and tubo-ovarian abscess. Infect Dis Clin North Am 2008;22:693-708.

8. Soper DE. Pelvic inflammatory disease. Obstet Gynecol 2010;116:419-28.

9. Daly JW. Pelvic abscess. En: Monif GRG, Baker DA, editores. Infectious diseases in obstetrics and gynecology. New York: The Parthenon Publishing Group; 2004. p. 894-902.

10. Mokdad C, Rozsnayi F, Delaunay F, Gregorczyk V, Auber M, Puscasiu L, et al. Surgical treatment of tubo-ovarian abscess occurring in deep endometriosis. Gynecol Obstet Fertil 2010;38:490-5.

11. Tanir HM, Hassa H, Ozalp S, Kaya M, Oge T. Pelvic abscess in intrauterine device users. Eur J Contracept Reprod Health Care 2005;10:15-8.

12. Hager WD, Eschenbach DA, Spence MR, Sweet RL. Criteria for diagnosis and grading of salpingitis. Obstet Gynecol 1983;61:113-4.

13. Pedowitz P, Bloomfield RD. Ruptured adnexal abscess (tuboovarian) with generalized peritonitis. Am J Obstet Gynecol 1964;88:721-9.

14. Kaplan AL, Jacobs WM, Ehresman JB. Aggressive management of pelvic abscess. Am J Obstet Gynecol 1967;98:482-7. 
15 Rosen M, Breitkopf D, Waud K. Tubo-ovarian abscess management options for women who desire fertility. Obstet Gynecol Surv 2009;64:681-9.

16. Rivlin ME. Conservative surgery for adnexal abscess. J Reprod Med 1985;30:726-30.

17. Landers DV, Sweet RL. Tubo-ovarian abscess: contemporary approach to management. Rev Infect Dis 1983;5:876-84.

18. Dellenbach P, Muller P, Philippe E. Infections utero-annexielles aigues. Encycl Med Chir Gynecol 1972;470:A10.

19. Granberg S, Gjelland K, Ekerhovd E. The management of pelvic abscess. Best Pract Res Clin Obstet Gynaecol 2009;23:667-78.

20. Henry-Suchet J, Soler A, Loffredo V. Laparoscopic treatment of tuboovarian abscesses. J Reprod Med 1984;29:579-82.
21. Henry-Suchet J. PID: clinical and laparoscopic aspects. Ann N Y Acad Sci 2000;900:301-8.

22. Reich H, McGlynn F. Laparoscopic treatment of tuboovarian and pelvic abscess. J Reprod Med 1987;32:747-52.

23. Raiga J, Denoix S, Canis M, Lebouedec G, Glowaczower E, Wattiez A, et al. Laparoscopic treatment of adnexal abscesses. A series of 39 patients. J Gynecol Obstet Biol Reprod (Paris) 1995;24:381-5.

24 Buchweitz O, Malik E, Kressin P, Meyhoefer-Malik A, Diedrich K. Laparoscopic management of tuboovarian abscesses: retrospective analysis of 60 cases. Surg Endosc 2000;14:948-50.

25. Yang CC, Chen P, Tseng JY, Wang PH. Advantages of open laparoscopic surgery over exploratory laparotomy in patients with tubo-ovarian abscess. J Am Assoc Gynecol Laparosc 2002;9:327-32.

\section{Conflicto de intereses: ninguno declarado}

